\title{
Infall in Protostellar Envelopes
}

\author{
Nagayoshi Ohashi \\ Institute of Astronomy $\&$ Astrophysics, Academia Sinica, P.O. Box \\ 23-141, Taipei 106, Taiwan, R.O.C.
}

\begin{abstract}
We summarize recent progress of observational studies of infall in protostellar envelopes, with great emphasis on results obtained using millimeter and submillimeter interferometers. Interferometric observations allow us to spatially resolve kinematical structures of protostellar envelopes, enabling us to detect infalling motions in the envelope directly. High angular resolution observations of infalling envelopes having compact disks sufficiently bright in continuum show inverse P-Cygni profiles, which are the least ambiguous evidence for infall. Observations of infalling envelopes using the Submillimeter Array (SMA) may allow us to study the innermost infalling envelopes, where infalling motions most probably transform to Keplerian motions, leading to formation of Keplerian or protoplanetary disks around protostars.
\end{abstract}

\section{Introduction}

It has been believed that low-mass stars are formed in dense cores through dynamical infall of the matter in the cores. Theoretical models of dynamically infalling cores have been developed earlier (e.g., Larson 1969; Penston 1969; Shu 1977.), while observations of infall did not make quick progress until early 1990's.

In order for us to observationally study infall, it is necessary to identify (candidate) protostars, on which significant amount of matter must accrete. Since protostars are very deeply embedded into parent molecular clouds, they are not detectable at optical wavelengths but are detectable at far-infrared wavelengths. Observations conducted by the Infrared Astronomical Satellite (IRAS) revealed the existence of many infrared point sources that have no optical counterparts in molecular clouds (Beichman et al. 1986). Because these infrared sources are optically invisible and much more deeply embedded in molecular cloud cores than T Tauri stars (i.e., optically visible pre-main-sequence starts), these sources have been regarded as protostar candidates. Whether or not these protostar candidates are "true" protostars, in the sense that they are objects accreting the bulk of the material in the surrounding envelopes, was not unambiguously demonstrated. Hence, many efforts have been made to obtain evidence for infall from protostar candidates. Specifically, a spectral signature of inward motion, called infall asymmetry, has been sought (e.g., Zhou 1992; Zhou et al. 1995): if the foreground infalling gas has lower excitation temperature than the background gas in a spherically infalling envelope, and if the foreground gas has sufficient optical depth, then the optically thick line will be skewed to the blue or be 
double-peak, with a stronger blue peak. Although this method has been widely accepted (e.g., Mardones et al. 1997; Gregersen et al. 1997), it is highly modeldependent, and in this sense, the infall asymmetry is still indirect evidence for infall. In addition, other motions such as outflow and rotation could possibly contaminate infall motions, which makes interpretation of infall asymmetry even more difficult. In fact, single dish observations of CS $J=2-1$ and 5-4 emission from B335 showing infall asymmetry was interpreted as infall (Zhou et al. 1993), while higher angular resolution observations of CS 5-4 from B335 suggested that the CS 5-4 emission is actually dominated by the outflowing gas (Wilner et al. 2000).

\section{Direct Imaging of Infalling Motions using Interferometers}

Interferometric observations at high angular resolution can spatially resolve velocity structures of molecular envelopes, which enables us to directly image the infalling material. In addition, high angular resolutions provided by interferometers allow us to distinguish infall motions from other motions such as outflow and rotation, which are sometimes confused in single-dish observations at lower angular resolutions. In fact, molecular envelopes have a flattened structure. Hence, blueshifted emission due to infall is observed from the far side of the envelope, and redshifted emission is observed from the near side. Thus infall is identified as a velocity gradient along the projected minor axis of the envelope. If rotation exists in an envelope, on the other hand, then it produces a velocity gradient along the major axis. If both infall and rotation exist in envelopes, then we see a velocity gradient along somewhere between the major axis and the minor axis of the envelope. In this approach, it is better for us to use optically thin lines rather than optically thick lines because optically thin lines can show velocity gradients more clearly without the self-absorption optically thick lines often show. With this strategy, infall in the envelope around HL Tau was first identified (Hayashi, Ohashi, \& Miyama 1993). After this first case, observations of infall toward more sources have been obtained (Ohashi et al. 1996, 1997a, 1997b; Saito et al. 1996; Yang et al. 1997; Momose et al. 1998; Park et al. 2000; Hirano et al. 2002), including a starless core (Ohashi et al. 1999).

Figure 1 shows an excellent example of interferometric images of an infalling envelope, which shows the above characteristics, obtained in $\mathrm{C}^{18} \mathrm{O} 1-0$ from L1551 IRS5 with the Nobeyama Millimeter Array (Momose et al. 1998). L1551 IRS5 is a well known protostar candidate in the Taurus star-forming regions $(\mathrm{d}=140 \mathrm{pc})$. The envelope around L1551 IRS5 has a clear disklike structure, $2400 \mathrm{AU} \times 1100 \mathrm{AU}$ in size, which is elongated in the direction perpendicular to the axis of the associated outflow (Fig. 1a). The southern half of the envelope is blueshifted, while its northern half is redshifted, as shown in the intensity weighted mean velocity map (Fig. 1b): the overall velocity gradient of the envelope is in the north-south direction, which is different from either the direction of the minor or major axis. This suggests that both infall and rotation exist in the envelope because pure infall yields a velocity gradient along the minor axis while pure rotation yields a gradient along the major axis.

Detailed velocity structures of infall and rotation can be examined by using position-velocity (PV) diagrams. The PV diagram along the major axis of the 

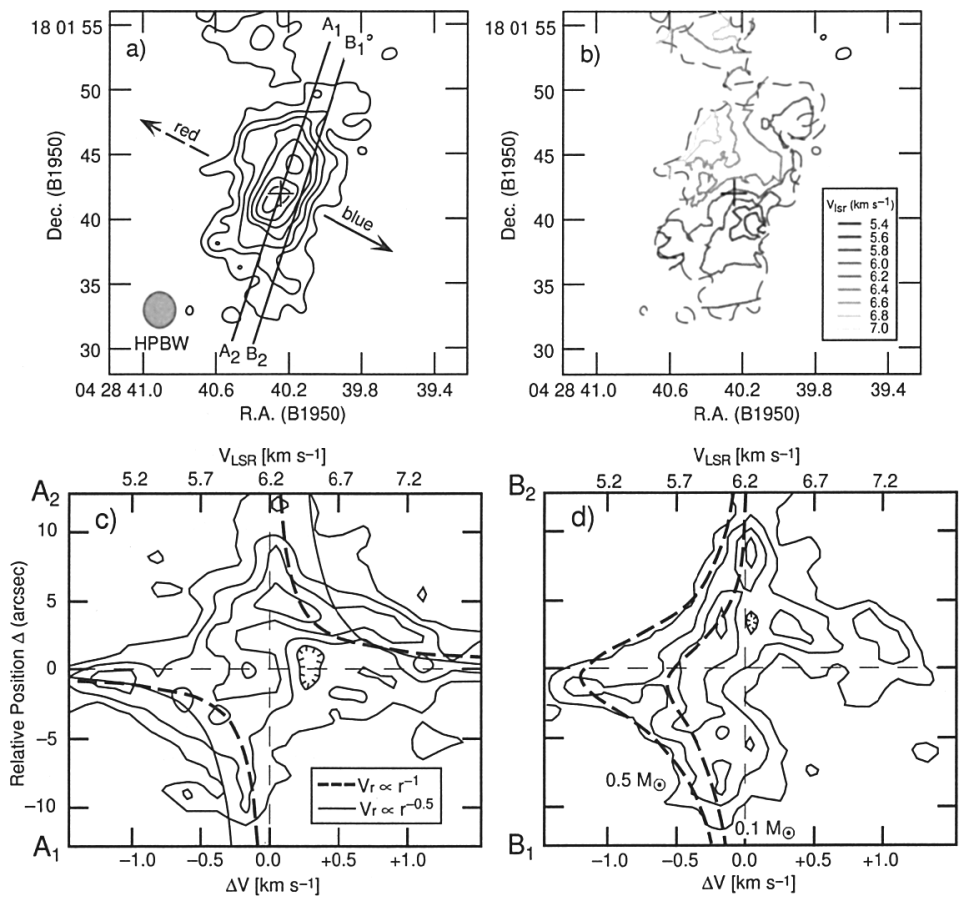

Figure 1. Interferometric $\mathrm{C}^{18} \mathrm{O}$ 1-0 maps of L1551 IRS5 (Momose et al. 1998). The contour spacing is $1.5 \sigma$, starting from $1.5 \sigma$ in (a), (c), and (d). (a) The total intensity map. The direction of the associated outflow is indicated by arrows, while the cross indicates the position of the central star. (b) The intensity-weighted mean velocity map. Bluer velocities are drawn in darker grey contours. The $1.5 \sigma$ contour of the total intensity is drawn in the dashed contour. (c) The PV diagram along the projected major axis of the $\mathrm{C}^{18} \mathrm{O}$ envelope [the line $\mathrm{A}_{1} \mathrm{~A}_{2}$ in (a)]. The dashed curves indicate the rotation curve with the velocity proportional to $r^{-1}$, while the thin solid curves show the Keplerian rotation yielded by a $0.15 \mathrm{M}_{\odot}$ central star. (d) The PV diagram along the cut $\mathrm{B}_{1} \mathrm{~B}_{2}$ in (a) offset by $1^{\prime \prime} 65$ southwest from the major axis. The two dashed curves indicate distribution of dynamical infall velocities; the inner curve is the infall around a $0.1 \mathrm{M}_{\odot}$ star, while the outer one is that around a $0.5 \mathrm{M}_{\odot}$ star. 
envelope (Fig. 1c) shows a velocity shift due to rotation. A remarkable feature is that the amount of velocity shift increases as the position approaches the central star, indicative of rotation velocity getting higher toward inner radii. On the other hand, the PV diagram along the cut offset by 1 ".65 southwest (the far-side of the envelope) from the major axis (Fig. 1d) shows mainly blueshifted emission getting much bluer as the position approaches the center (Fig. 1d). This feature can be explained by infalling motions, which accelerate toward the center. In addition, the most blueshifted emission is not located at the center but is slightly shifted to the southeast $\left(\Delta<0^{\prime \prime}\right)$. This deviation is naturally explained by rotation.

A simple analysis on the assumption of a geometrically thin envelope shows that the radial dependence of the rotation velocity is consistent with $r^{-1}$, which is the case of angular momentum conservation, while the radial dependence of the infall velocity is consistent with the case of dynamical infall $\left(\propto r^{-0.5}\right)$ yielded by a $0.1-0.5 \mathrm{M}_{\odot}$ central star. The infall and rotation velocities at $r=700 \mathrm{AU}$ are $0.5 \mathrm{~km} \mathrm{~s}^{-1}$ and $0.24 \mathrm{~km} \mathrm{~s}^{-1}$, respectively. The infall rate was estimated to be $6.4 \times 10^{-6} \mathrm{M}_{\odot} \mathrm{yr}^{-1}$. Note that there is an additional redshifted emission in Fig. 1d. This emission originates from infalling gas in the near-side of the envelope, which is observable when the envelope is geometrically thick and nearly edge-on with respect to observers.

\section{Inverse $\mathbf{P}$ Cygni line profile}

Not only optically thin lines but also optically thick lines are still useful to probe infalling motions with interferometers. Infall asymmetry was observed using interferometers toward some protostar candidates (B335, Chandler and Sargent 1993; Choi et al. 1999, L1527 Zhou et al. 1996; Wilner et al. 1997). We have to be careful, however, when we interpret infall asymmetry observed using interferometers because line profiles obtained interferometers often show "dips" at around systemic velocities of the envelopes because of resolving out extended structures. These dips could artificially produce infall asymmetry.

In the case where envelopes contain compact disks sufficiently bright in continuum, optically thick lines are even more useful to probe infall: in such a case, the front side of the envelope will appear in absorption while the back side of the envelope will appear in emission, producing an inverse P Cygni line profile. Choi (2002) simulated line profile of moderately optically thick molecular transitions from an infalling envelope, and found that there is yet another absorption dip, in addition to an absorption dip near zero velocity, on the redshifted side. This absorption is caused by the very inner part of the infall region, and can be more obvious with a compact, strong continuum emission and with observations at high angular resolutions.

Detections of inverse P Cygni line profile have been made primarily toward high- mass young stellar objects, where the absorption profiles were seen against the continuum emission of bright H II regions (e.g., Zhang, Ho, and Ohashi 1998). On the other hand, toward low-mass young stellar objects, detections of inverse $\mathrm{P}$ Cygni line profile against continuum emission have been very rare: only NGC $13334 \mathrm{~A}$ and $4 \mathrm{~B}$ have showed inverse $\mathrm{P}$ Cygni line profile in $\mathrm{H}_{2} \mathrm{CO} 3_{12}-2_{11}$ (Di Francesco et al. 2001). Although the redshifted absorption could arise from 
a foreground cloud that just happens to be redshifted, a higher transition line such as $\mathrm{H}_{2} \mathrm{CO} 3_{12}-2_{11}$ more likely arises from warmer gas associated with central protostars.

\section{Observations of infall using the Submillimeter Array}

As shown in previous sections, interferometric observations at millimeter wavelengths with high angular resolutions are a good approach to study infall in protostellar envelopes. However, there are still several important questions, which previous interferometric observations at millimeter wavelengths have not addressed;

- What happens to infalling motions at the innermost envelopes?

- Do infalling motions settle down to Kepler rotation? If yes, how does it happen?

- Are there any shocks associated with the transition from infall motions to Kepler motions?

Since angular momentum of the infalling material is conserved and rotation velocity increases as $r^{-1}$, infalling material cannot go inward any more and will be rotationally supported at some point, where the rotation velocity gets comparable to infalling velocity. The size scale where this transition occurs is estimated to be of order $100 \mathrm{AU}$. No interferometric observations, however, have demonstrated this transition. Interferometer at submillimeter wavelengths would be able to reveal what is going on at the innermost infalling envelopes because emission at submillimeter wavelengths could traces only the innermost envelope with less contamination of emission arising from the extended envelope.

The Submillimeter Array (SMA) is the first imaging array at submillimeter wavelengths, which has been constructed at the top of Mauna Kea, Hawaii, as a collaborative project of the Smithonian Astrophysical Observatory (SAO) and the Academia Sinica Institute of Astronomy and Astrophysics of Taiwan (ASIAA). Although the construction is not completed at this time (October 2003), the partly completed array has been used to carry out initial scientific projects (see Zhang et al. in this volume for more results of the SMA initial science). As a representative of low-mass protostars, L1551 IRS5 was observed in CS 7-6 using the SMA (Takakuwa et al. in prep.). As was shown in the section 2, since the envelope surrounding L1551 IRS5 shows infalling motions, this is a good target to study the innermost infalling envelope at submillimeter wavelengths.

Figure 2 shows CS the 7-6 total intensity map of L1551 IRS5, obtained using the SMA, showing that a CS condensation, centered at the position of L1551 IRS5, is detected with the SMA. For a comparison, the $\mathrm{C}^{18} \mathrm{O} 1-0$ total intensity contour map (Momose et al. 1998), obtained using the Nobeyama Millimeter Array, is superimposed. As shown in the section 2, the $\mathrm{C}^{18} \mathrm{O}$ envelope shows infalling motions with slow rotation. It is very clear that the CS condensation is much more compact than the $\mathrm{C}^{18} \mathrm{O}$ envelope. The size of the CS condensation is $\sim 400 \mathrm{AU}$. Since CS $7-6$ line selectively traces higher-density $\left(Z 10^{7} \mathrm{~cm}^{-3}\right)$ 


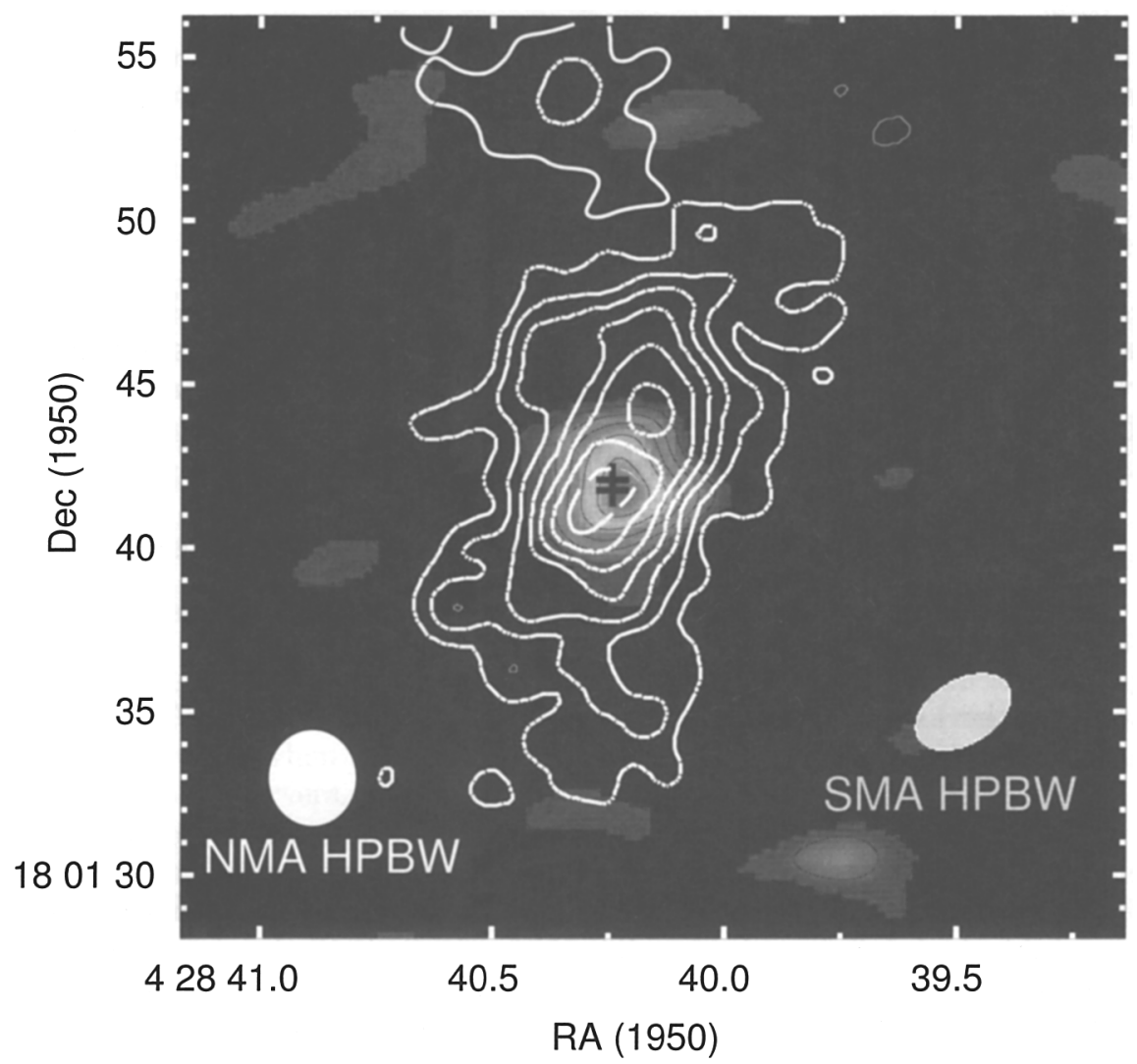

Figure 2. CS 7-6 total intensity map of L1551 IRS5, obtained with the SMA, is shown in grey scales. The angular resolution is $3 . ! 2 \times$ 2 !' 1 . Two crosses show the positions of the central stars (binary). For a comparison, the $\mathrm{C}^{18} \mathrm{O} 1-0$ total intensity map shown in Fig. 1 (Momose et al. 1998) is also drawn in white contours.

and higher-temperature $(z 40 \mathrm{~K})$ regions, the detected CS condensation is most probably the innermost envelope of L1551 IRS5. It is also interesting to note that the CS condensation does not clearly show an elongated structure, which is obvious in the $\mathrm{C}^{18} \mathrm{O}$ envelope.

The mean velocity map of the CS condensation shows that there is a velocity gradient along the direction perpendicular to the associated outflow, and the sense of the gradient is the same as that seen in the $\mathrm{C}^{18} \mathrm{O}$ envelope. Figure 3 shows position-velocity (PV) diagrams of the CS condensation and the $\mathrm{C}^{18} \mathrm{O}$ envelope, with the same cuts along the major axis of the $\mathrm{C}^{18} \mathrm{O}$ envelope. The PV diagram of the CS condensation shows that there are two velocity components, one at a low velocity and the other at a high velocity, located very close to the central star ( $\lesssim 1^{\prime \prime}$ from the central star). The CS high velocity component has counter parts in the $\mathrm{C}^{18} \mathrm{O} \mathrm{PV}$ diagram, and it may be explained as a part of the rotation motions observed in the $\mathrm{C}^{18} \mathrm{O}$ envelope, as indicated with thick 


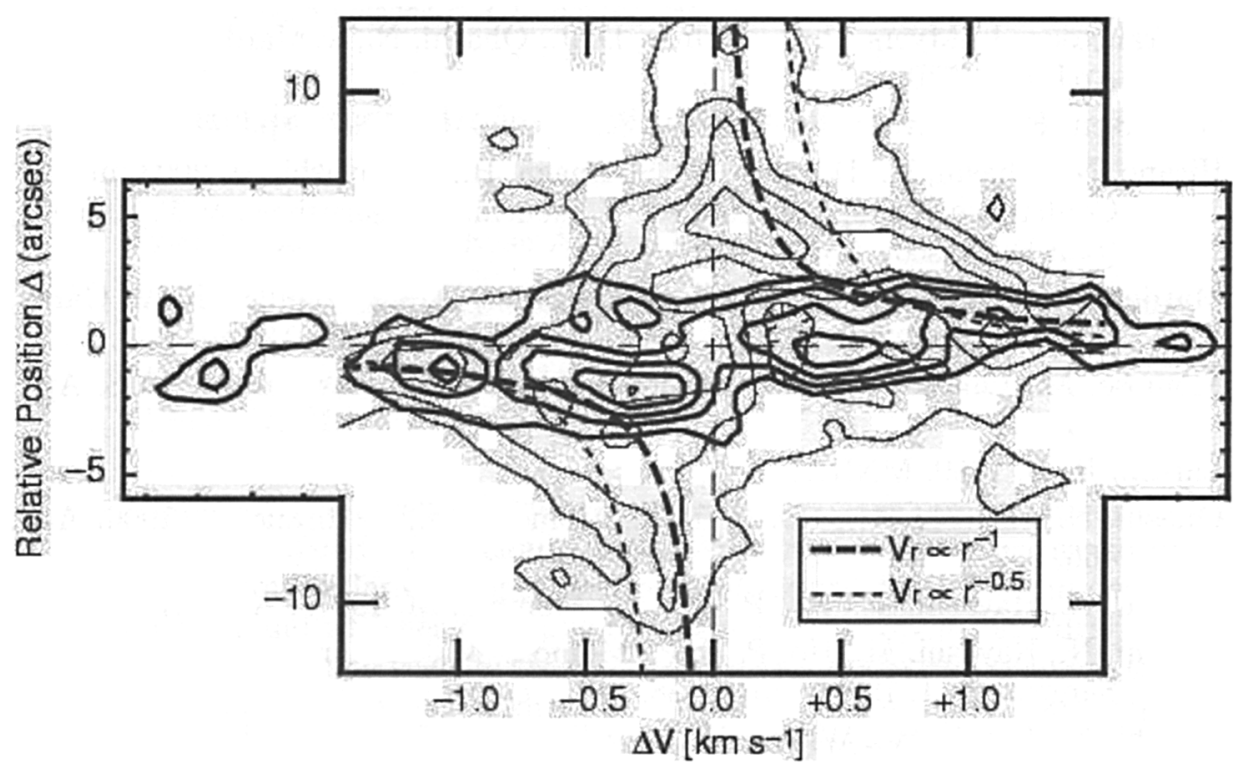

Figure 3. PV diagrams of the CS condensation (black contours) and the $\mathrm{C}^{18} \mathrm{O}$ envelope (grey contours). Both diagrams are cuts along the major axis of the $\mathrm{C}^{18} \mathrm{O}$ envelope.

dashed curves, or could be explained as Keplerian rotation, as indicated with thin dashed curves. On the other hand, the low velocity components of the $\mathrm{CS}$ do not have any counter parts in the $\mathrm{C}^{18} \mathrm{O} \mathrm{PV}$ diagram, and cannot be explained as a part of the rotation motions observed in $\mathrm{C}^{18} \mathrm{O}$. There is no clear interpretation of this low velocity component so far.

One must note that there is significant missing flux of the CS 7-6 emission in this SMA observation. In such a case, we may actually have resolved out extended CS emission having lower velocities, and as a result, only low velocity components close to the central star remain detectable. It is definitely important to conduct further observations using the SMA to see more details of the kinematics of the innermost envelope around L1551 IRS5.

Acknowledgments. I thank Dr. Takakuwa, who provided me with figures of L1551 IRS5, which were unpublished yet. I also thank the NSC, which supported my travel to participate in the symposium.

\section{References}

Beichiman, C. A., Myers, P. C., Emerson, J. P., Harris, S., Mathieu, R., Benson, P. J., \& Jennings, R. E. 1986, ApJ, 307, 337

Chandler, J. C., \& Sargent, A. I. 1993, ApJ, 414, L29

Choi, M. 2002, ApJ, 575, 900

Choi, M., Panis, J-F., \& Evans, N. J., II. 1999, ApJS, 122, 519 
Di Francesco, J., Myers, P. C., Wilner, D. J., Ohashi, N., \& Mardones, D. 2001, ApJ, 562, 770

Gregersen, E. M., Evans, N. J., Zhou, S., \& Choi, M. 1997, ApJ, 484, 256

Hirano, N., Ohashi, N., Dobashi, K., Shinnaga, H., \& Hayashi, M. 2003, in ASP Conference Series, Vol. 289, ed. S. Ikeuchi, J. Hearnshaw, \& T. Hanawa, (San Francisco: ASP), 141

Mardones, D., Myers, P. C., Tafalla, M., Wilner, D. J., Bachiller, R., \& Garay, G. 1997, ApJ, 489, 719

Momose, M., Ohashi, N., Kawabe, R., Nakano, T., \& Hayashi, M. 1998, ApJ, 504, 314

Larson, R. B. 1969, MNRAS, 145, 271

Ohashi, N., Hayashi, M., Ho, P. T. P., Momose, M., \& Hirano, N. 1996, ApJ, 466, 957

Ohashi, N., Hayashi, M., Ho, P. T. P., \& Momose, M. 1997a, ApJ, 475, 211

Ohashi, N., Hayashi, M., Ho, P. T. P., Momose, M., Tamura, M., Hirano, N., \& Sargent, A. I. 1997b, ApJ, 488, 317

Ohashi, N., Lee, S.-W., Wilner, D. J., \& Hayashi, M. 1999, ApJ, 518, L41

Park, Y.-S., Panis, J.-F., Ohashi, N., Choi, M., \& Minh, Y. C. 2000, ApJ, 542, 344

Penston, M. V. 1969, MNRAS, 144, 425

Shu, F. H. 1977, ApJ, 214, 488

Wilner, D. J., Mardones, D., \& Myers, P. C. 1997, in AIP Conf. Proc., 393, Star Formation Near and Far, ed. S. S. Holt \& L. G. Mundy (New York: AIP), 109

Wilner, D. J., Myers, P. C., Mardones, D., \& Tafalla, M. 2000, ApJ, 544, L69

Yang, J., Ohashi, N., Yan, J., Liu, C., Kaifu, N., \& Kimura, H. 1997, ApJ, 475, 683

Zhang, Q., Ho, P. T. P., \& Ohashi, N. 1998, ApJ, 494, 636

Zhou, S. 1992, ApJ, 394, 204

Zhou, S. 1995, ApJ, 442, 685

Zhou, S., Evans, N. J., II., Kompe, C., \& Walmsley, C. M. 1993, ApJ, 404, 232

Zhou, S., Evans, N. J., II, \& Wang, Y. 1996, ApJ, 466, 296 